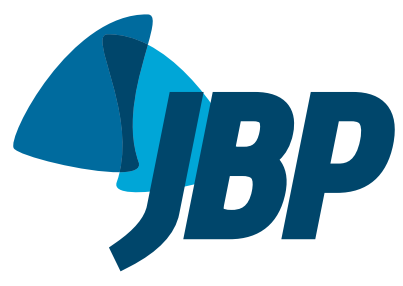

\title{
Obstructive sleep apnea related to rapid-eye- movement or non-rapid-eye-movement sleep: comparison of demographic, anthropometric, and polysomnographic features
}

\author{
Aysel Sunnetcioglu1, Bunyamin Sertogullarından1, Bulent Ozbay², \\ Hulya Gunbatar ${ }^{1}$, Selami Ekin ${ }^{1}$
}

1. Pulmonology Department, Yuzuncu Yil University School of Medicine, Van, Turkey.

2. Pulmonology Department, Muğla Sıtkı Koçman University School of Medicine, Muğla, Turkey.

Submitted: 24 January 2015.

Accepted: 08 August 2015

Study carried out in the Pulmonology Department, Yuzuncu Yil University School of Medicine, Van, Turkey; and in the Pulmonology Department, Muğla Sıtkı Koçman University School of Medicine, Muğla, Turkey.

\begin{abstract}
Objective: To determine whether there are significant differences between rapid-eyemovement (REM)-related obstructive sleep apnea (OSA) and non-REM (NREM)-related OSA, in terms of the demographic, anthropometric, and polysomnographic characteristics of the subjects. Methods: This was a retrospective study of 110 patients ( 75 males) with either REM-related OSA ( $n=58$ ) or NREM-related OSA $(n=52)$. To define REM-related and NREM-related OSA, we used a previously established criterion, based on the apneahypopnea index (AHI): AHI-REM/AHI-NREM ratio > 2 and $\leq 2$, respectively. Results: The mean age of the patients with REM-related OSA was $49.5 \pm 11.9$ years, whereas that of the patients with NREM-related OSA was $49.2 \pm 12.6$ years. The overall mean AHI (all sleep stages combined) was significantly higher in the NREM-related OSA group than in the REM-related OSA group (38.6 \pm 28.2 vs. $14.8 \pm 9.2 ; p<0.05)$. The mean AHI in the supine position (s-AHI) was also significantly higher in the NREM-related OSA group than in the REM-related OSA group $(49.0 \pm 34.3$ vs. $18.8 \pm 14.9 ; p<0.0001)$. In the NREM-related OSA group, the s-AHI was higher among the men. In both groups, oxygen desaturation was more severe among the women. We found that REM-related OSA was more common among the patients with mild-to-moderate OSA, whereas NREMrelated OSA was more common among those with severe OSA. Conclusions: We found that the severity of NREM-related OSA was associated mainly with s-AHI. Our findings suggest that the s-AHI has a more significant effect on the severity of OSA than does the AHI-REM. When interpreting OSA severity and choosing among treatment modalities, physicians should take into consideration the sleep stage and the sleep posture.
\end{abstract}

Keywords: Sleep, REM; Sleep stages; Sleep apnea, obstructive; Apnea; Sleep apnea syndromes.

\section{INTRODUCTION}

Obstructive sleep apnea (OSA) is a common sleep disorder, characterized by recurrent episodes of complete or partial upper airway collapse, accompanied by intermittent hypoxemia and recurrent arousals from sleep. Whereas upper airway collapse can occur during rapid-eye-movement (REM) and non-REM (NREM) sleep, the withdrawal of excitatory noradrenergic and serotonergic inputs to the upper airway motor neurons during REM sleep further reduces the pharyngeal muscle activity and substantially increases the propensity for such collapse. (1) Therefore, in patients with OSA, REM sleep is typically associated with an increased frequency of obstructive events that are often prolonged and are accompanied by severe oxygen desaturation. In some patients, respiratory events occur predominantly during REM sleep. ${ }^{(1-4)}$ A commonly used diagnostic criterion is the ratio between the apnea-hypopnea index (AHI) during REM sleep and the AHI during NREM sleep (the AHI-REM/AHI-NREM ratio), an AHI-REM/AHI-NREM ratio $>2$ indicating a predominance of disordered breathing during REM sleep, or REM-related OSA. ${ }^{(5)}$ The reported prevalence of REM-related OSA in clinical studies varies widely, ranging from $10 \%$ to $36 \%$. $^{(1-4)}$ That variability is due, in part, to differences in sample characteristics and in the definition of REM-related OSA. ${ }^{(2)}$ It is well known that OSA is more common in the elderly, in males, in individuals with a high BMI, and in individuals who sleep in the supine position. ${ }^{(4,6)}$ However, REM-related OSA is reported to occur more commonly in younger individuals, women, children, and patients with mild or moderate OSA. ${ }^{(2,5,7-9)}$ The main aim of our study was to compare patients with REM-related OSA and patients with NREM-related OSA, in terms of their demographic, anthropometric, and polysomnography characteristics.

\section{METHODS}

This was a retrospective study of 110 patients (58 with REM-related OSA and 52 with NREM-related OSA) who underwent polysomnography in a sleep laboratory operated by the Pulmonology Department of the Yuzuncu Yil University School of Medicine, in the city of Van, Turkey, between January of 2013 and March of 2014. Patients with a sleep efficiency $<40 \%$ were excluded, 
as were those with an AHI $<5$ events/h, those in whom REM sleep accounted for $<15 \%$ of the total sleep time, and those who were under 15 years of age. For all patients, the AHI was calculated for total sleep time, for REM sleep (AHI-REM), and for NREM sleep (AHI-NREM), as well as for sleep in the supine position (s-AHI) and sleep in the lateral position (lateral AHI, right-hip and left-hip). We also collected the following data: age; gender; level of daytime sleepiness; BMI; neck circumference; total sleep time; REM sleep and NREM sleep, as percentages of the total sleep time; the mean oxygen desaturation time; and the minimum $\mathrm{SaO}_{2}$. We then attempted to determine whether any of those parameters differed between the REM-related OSA and NREM-related OSA groups.

\section{Polysomnography}

We performed polysomnography using a 16-channel polysomnograph (Embla; Medcare Flaga, Reykjavik, Iceland), with continuous monitoring by a technician. The system consists of four electroencephalography channels, two electrooculography channels, tibial/ submental electromyography, and electrocardiography, as well as monitoring of oronasal airflow, thoracic movements, abdominal movements, $\mathrm{SaO}_{2}$, and body position. Polysomnographic recordings were manually interpreted over 30-s intervals in accordance with the guidelines established by the American Academy of Sleep Medicine. ${ }^{(10)}$ Apnea was defined as the complete cessation of airflow for more than $10 \mathrm{~s}$. Hypopnea was defined as a $\geq 30 \%$ reduction in respiratory airflow lasting for more than $10 \mathrm{~s}$, accompanied by $\mathrm{a} \geq 4 \%$ decrease in $\mathrm{SaO}_{2}$. Arousal was defined as a sudden change in the electroencephalography frequency, consisting of alpha and theta activity or waveforms with frequencies $>16 \mathrm{~Hz}$ (although not sleep spindles) and a duration of 3-15 s. Respiratory effort-related arousals occur when there is a sequence of breaths that last for at least $10 \mathrm{~s}$, characterized by increased respiratory effort or flattening of the nasal pressure waveform, followed by arousal from sleep, which does not meet the criteria for an apnea or hypopnea event. ${ }^{(11)}$ We determined the average overall AHI, expressed as the number of events per hour of sleep. For each patient, OSA was classified as mild (AHI, 5-15 events/h), moderate (AHI, 16-30 events/h), or severe (AHI, > 30 events/h).

In accordance with a previous report, ${ }^{(5)}$ we identified respiratory disorders predominantly restricted to REM sleep by calculating the AHI-REM/AHI-NREM ratio. Patients with an AHI-REM/AHI-NREM ratio $>2$ were categorized as having REM-related OSA, whereas those with an AHI-REM/AHI-NREM ratio $\leq 2$ were categorized as having NREM-related OSA. ${ }^{(5)}$ The subjective level of daytime sleepiness was quantified with a self-report questionnaire, the Epworth Sleepiness Scale. ${ }^{(12)}$

\section{Statistical analysis}

The results are expressed as mean \pm standard deviation. Student's t-tests were used in order to compare the means of two independent variables such as gender and group (REM-related OSA vs. NREM-related OSA). Age was included in the model as a covariate to remove extraneous influences from the dependent variable, thus decreasing the variance within the group, and to adjust the means of the groups. Chi-square and Fisher's exact tests were used in order to test the independence of categorical variables. Pairwise Pearson correlation tests were carried out in order to estimate the linear relationship between the characteristics. All statistical calculations were performed using the Statistical Analysis System software, version 9.3 (SAS Institute, Cary, NC, USA). Values of $p<0.05$ were considered statistically significant.

\section{RESULTS}

Of the 110 OSA patients evaluated, 58 met the criteria for REM-related OSA, whereas 52 met the criteria for NREM-related OSA. The mean age of the patients with REM-related OSA was $49.5 \pm 11.9$ years, compared with $49.2 \pm 12.6$ years for those with NREM-related OSA (Table 1). There was a predominance of males in our study sample (68\%), and the proportion of males was higher in the NREM-related OSA group than in the REM-related OSA group (84.6\% vs. $53.4 \%$; Table 1 ).

There was no statistically significant difference between the REM-related and NREM-related OSA groups in terms of the mean BMI $\left(33.3 \pm 5.7 \mathrm{~kg} / \mathrm{m}^{2}\right.$ vs. 32.2 $\left.\pm 5.4 \mathrm{~kg} / \mathrm{m}^{2} ; \mathrm{p}=0.97\right)$. As can be seen in Figure 1 , the AHI value correlated positively with the BMI in the REM-related OSA group ( $r=0.343 ; p<0.01$ ). In the NREM-related OSA group, the mean BMI was significantly higher in the females than in the males $(39.0 \pm 4.2$ vs. $30.9 \pm 4.6 ; p<0.05)$. There was no significant difference in AHI between the genders, in either group (Table 2). There was also no statistically significant difference between the two groups in terms of the mean Epworth Sleepiness Scale score (15.4 \pm 5.3 vs. $15.6 \pm 6.8 ; p=0.94$ ).

The mean AHI was significantly lower in the REM-related OSA group than in the NREM-related OSA group $(14.8 \pm 9.2$ vs. $38.6 \pm 28.2 ; p<0.05)$. In the REM-related OSA group, the OSA was classified as mild in $36(62.1 \%)$ of the 58 patients, moderate in 16 $(27.6 \%)$, and severe in $6(10.3 \%)$, compared with 12 $(23.1 \%), 15(28.9 \%)$, and $25(48.1 \%)$, respectively, for the NREM-related group. In the REM-related OSA group, the AHI correlated positively with age $(r=0.344 ; p<0.05)$ and with BMI $(r=0.343 ; p<$ $0.05)$. The AHI also correlated positively with BMI in the NREM-related OSA group, although the difference was not significant.

The supine, right-lateral, and left-lateral AHI values were higher in the NREM-related OSA group than in the REM-related OSA group (Table 1). As can be seen in Figure 2, the AHI correlated positively with s-AHI in the NREM-related OSA group $(r=0.707 ; p<0.01)$. In NREM-related OSA, the s-AHI was higher among 
Table 1. Demographic, anthropometric, and polysomnographic features of patients with obstructive sleep apnea. ${ }^{a}$

\begin{tabular}{|c|c|c|c|}
\hline \multirow[t]{2}{*}{ Variables } & \multicolumn{2}{|c|}{ Type of OSA } & \multirow[t]{2}{*}{$\mathbf{p}$} \\
\hline & $\begin{array}{c}\text { REM-related } \\
(\mathrm{n}=58)\end{array}$ & $\begin{array}{l}\text { NREM-related } \\
\qquad(\mathrm{n}=52)\end{array}$ & \\
\hline Age, years & $49.5 \pm 11.9$ & $49.2 \pm 12.6$ & $>0.05$ \\
\hline Males $^{\mathrm{b}}$ & $31(53.4)$ & $44(84.6)$ & $<0.05$ \\
\hline $\mathrm{BMI}, \mathrm{kg} / \mathrm{m}^{2}$ & $33.3 \pm 5.7$ & $32.2 \pm 5.4$ & 0.974 \\
\hline Neck circumference, $\mathrm{cm}$ & $38.1 \pm 3.4$ & $39.6 \pm 3.3$ & 0.589 \\
\hline ESS score & $15.4 \pm 5.3$ & $15.6 \pm 6.8$ & 0.943 \\
\hline TST, min & $348.1 \pm 63.3$ & $344.2 \pm 63.8$ & 0.931 \\
\hline WASO, \% & $89.9 \pm 55.2$ & $82.8 \pm 52.7$ & 0.656 \\
\hline Arousals/h & $7.16 \pm 11.1$ & $7.71 \pm 11.7$ & 0.756 \\
\hline RERAs & $6.79 \pm 5.50$ & $8.45 \pm 6.29$ & 0.343 \\
\hline Oxygen desaturation time, ${ }^{c}$ min & $48.5 \pm 58.0$ & $46.3 \pm 44.3$ & 0.990 \\
\hline Minimum $\mathrm{SaO}_{2}, \%$ & $77.0 \pm 9.0$ & $74.1 \pm 16.3$ & 0.102 \\
\hline $\mathrm{SaO}_{2}, \%$ & $89.8 \pm 3.7$ & $88.3 \pm 4.4$ & 0.017 \\
\hline AHI, events/h & $14.8 \pm 9.23$ & $38.6 \pm 28.2$ & $<0.0001$ \\
\hline Supine AHI, events/h & $18.8 \pm 14.9$ & $49.0 \pm 34.3$ & $<0.0001$ \\
\hline Left-lateral AHI, events/h & $15.8 \pm 13.8$ & $27.0 \pm 32.8$ & 0.031 \\
\hline Right-lateral AHI, events/h & $14.0 \pm 15.2$ & $29.8 \pm 32.6$ & 0.001 \\
\hline Total apnea events & $9.4 \pm 16.8$ & $19.2 \pm 25.1$ & 0.016 \\
\hline Total hypopnea events & $24.8 \pm 12.4$ & $23.0 \pm 17.4$ & 0.524 \\
\hline REM sleep, \% & $17.4 \pm 6.3$ & $17.3 \pm 16.7$ & 0.874 \\
\hline NREM sleep, \% & $34.4 \pm 19.5$ & $42.4 \pm 29.0$ & 0.851 \\
\hline Comorbid disorders $^{\mathrm{b}}$ & $13(22.4)$ & $12(23.1)$ & 0.934 \\
\hline
\end{tabular}

OSA: obstructive sleep apnea; REM: rapid-eye-movement (sleep); NREM: non-rapid-eye-movement (sleep); ESS: Epworth Sleepiness Scale; TST: total sleep time; WASO: wake after sleep onset; RERAs: respiratory effort-related

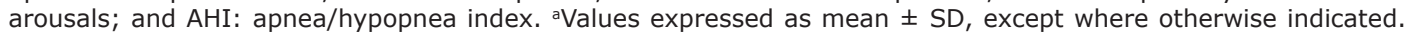
bValues expressed as $\mathrm{n}(\%) .{ }^{\mathrm{c}} \mathrm{SaO}_{2}<90 \%$.

men, whereas the right- and left-lateral AHI were both higher among women.

There were no differences between the REM-related and NREM-related OSA groups in terms of the wake after sleep onset and arousal values (Table 1). However, in the NREM-related OSA group, the wake after sleep onset value was significantly lower among the men than among the women (67.7 \pm 39.9 vs. $99.2 \pm 60.0$; $\mathrm{p}=0.027$; Table 2).

There was no significant difference between the REM-related and NREM-related OSA groups in terms of the mean oxygen desaturation time (48.5 \pm 58.0 vs. $46.3 \pm 44.3 ; p=0.990$ ). However, oxygen desaturation was more severe among the women than among the men, in both groups (Table 2). We also found that, in REM-related OSA, oxygen desaturation correlated positively with age $(r=0.355 ; p<0.05)$ and with BMI $(r=0.287 ; p<0.05)$.

There were no differences between the two groups with regard to comorbidities (Table 1 ). In the REM-related and NREM-related OSA groups, 13 and 12 patients, respectively, had one or more comorbidities, including COPD (in 5 and 4 patients, respectively), coronary artery disease (in 2 NREM-related OSA group patients), hypertension (in 7 and 2 patients, respectively), and diabetes mellitus (in 4 and 3 patients, respectively). As expected (given the lack of any differences in terms of comorbidities), there were also no differences between the two groups with regard to the medications taken by the patients.

\section{DISCUSSION}

In the present study, severe OSA was more common among the patients with NREM-related OSA than among those with REM-related OSA and was found to be associated mainly with the s-AHI. We also found that the prevalence of REM-related OSA was higher among the female patients, and that the female patients with REM-related OSA were younger and less obese than were those with NREM-related OSA.

During sleep, the most pronounced decrease in muscle tone typically occurs during REM sleep, causing atony, and the loss of tone in the dilator muscles makes it more likely that disordered breathing will occur. Such events, which occur during the night, can be associated with sleeping in the supine position or with REM sleep. Punjabi et al. found that the REM-AHI was higher than the NREM-AHI only in patients with an AHI of $<30$ events/h. ${ }^{(13)}$ In another study, similar to the present study, REM-related OSA was found to be more common in patients with moderate OSA than in those with severe OSA. ${ }^{(14)}$ That is in agreement with our findings that the AHI values were lower in the patients with REM-related OSA than in those with NREM-related OSA (the initial phase of REM-related OSA in the literature), ${ }^{(15)}$ and that the prevalence of 
Table 2. Features of patients with obstructive sleep apnea, by predominant sleep stage and by gender. ${ }^{\mathrm{a}}$

\begin{tabular}{|c|c|c|c|c|c|c|}
\hline \multirow[t]{3}{*}{ Variables } & \multicolumn{6}{|c|}{ Type of obstructive sleep apnea } \\
\hline & \multicolumn{3}{|c|}{ REM-related } & \multicolumn{3}{|c|}{ NREM-related } \\
\hline & Females & Males & $\mathbf{p}$ & Females & Males & $\mathbf{p}$ \\
\hline Age, years & $53.5 \pm 10.3$ & $46.0 \pm 12.3$ & 0.0166 & $62.3 \pm 12.7$ & $46.8 \pm 11.2$ & 0.0009 \\
\hline $\mathrm{BMI}, \mathrm{kg} / \mathrm{m}^{2}$ & $35.1 \pm 5.8$ & $31.8 \pm 5.3$ & 0.0274 & $39.0 \pm 4.2$ & $30.9 \pm 4.6$ & $<0.0001$ \\
\hline Neck circumference, $\mathrm{cm}$ & $36.0 \pm 2.7$ & $39.9 \pm 2.9$ & $<0.0001$ & $37.2 \pm 2.3$ & $40.0 \pm 3.3$ & 0.026 \\
\hline ESS score & $15.4 \pm 5.1$ & $15.4 \pm 5.5$ & 0.9752 & $16.8 \pm 6.0$ & $15.4 \pm 6.1$ & 0.546 \\
\hline TST, min & $355.6 \pm 63.7$ & $341.5 \pm 63.2$ & 0.4103 & $314.8 \pm 50.8$ & $349.8 \pm 65.8$ & 0.157 \\
\hline WASO, \% & $94,7 \pm 63.6$ & $89,3 \pm 55.0$ & 0.840 & $99,2 \pm 60.0$ & $67,7 \pm 39.9$ & 0.027 \\
\hline Arousals/h & $7,36 \pm 6.35$ & $7,14 \pm 11.6$ & 0.968 & $9,94 \pm 11.3$ & $5,80 \pm 11.3$ & 0.210 \\
\hline RERAs & $7,17 \pm 5.52$ & $6,71 \pm 5.59$ & 0.858 & $8,88 \pm 5.70$ & $7,83 \pm 7,27$ & 0.667 \\
\hline Oxygen desaturation time, ${ }^{\mathrm{b}} \min$ & $61.4 \pm 64.7$ & $37.4 \pm 49.8$ & 0.1175 & $59.6 \pm 34.6$ & $43.9 \pm 45.8$ & 0.360 \\
\hline Minimum $\mathrm{SaO}_{2}, \%$ & $74.8 \pm 9.8$ & $78.8 \pm 7.9$ & 0.0942 & $69.0 \pm 12.7$ & $75.0 \pm 13.7$ & 0.251 \\
\hline $\mathrm{SaO}_{2}, \%$ & $89.0 \pm 4.3$ & $90.5 \pm 2.9$ & 0.1293 & $85.6 \pm 5.7$ & $88.7 \pm 4.0$ & 0.068 \\
\hline $\mathrm{AHI}$, events/h & $15.5 \pm 9.2$ & $14.2 \pm 9.3$ & 0.5960 & $39.7 \pm 39.0$ & $38.4 \pm 26.3$ & 0.908 \\
\hline Supine AHI, events/h & $17.7 \pm 13.7$ & $19.6 \pm 16.2$ & 0.6806 & $22.0 \pm 24.2$ & $53.4 \pm 33.9$ & 0.023 \\
\hline Right-lateral AHI, events/h & $17.7 \pm 18.5$ & $11.4 \pm 11.9$ & 0.1508 & $40.0 \pm 50.2$ & $27.9 \pm 28.9$ & 0.374 \\
\hline Total apnea events & $10.7 \pm 22.1$ & $8.3 \pm 10.7$ & 0.5952 & $18.0 \pm 30.5$ & $19.4 \pm 24.4$ & 0.883 \\
\hline Total hypopnea events & $25.4 \pm 12.6$ & $24.2 \pm 12.5$ & 0.7239 & $24.2 \pm 9.9$ & $22.8 \pm 18.5$ & 0.844 \\
\hline REM sleep, \% & $18.3 \pm 7.3$ & $16.6 \pm 4.7$ & 0.2945 & $16.2 \pm 7.8$ & $17.3 \pm 6.6$ & 0.637 \\
\hline NREM sleep, $\%$ & $81.4 \pm 7.6$ & $83.0 \pm 5.5$ & 0.3647 & $83.7 \pm 7.8$ & $82.6 \pm 6.8$ & 0.704 \\
\hline
\end{tabular}

REM: rapid-eye-movement (sleep); NREM: non-rapid-eye-movement (sleep); ESS: Epworth Sleepiness Scale; TST: total sleep time; WASO: wake after sleep onset; RERAs: respiratory effort-related arousals; and AHI: apnea/

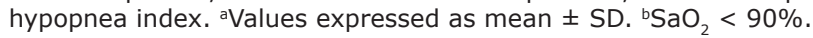

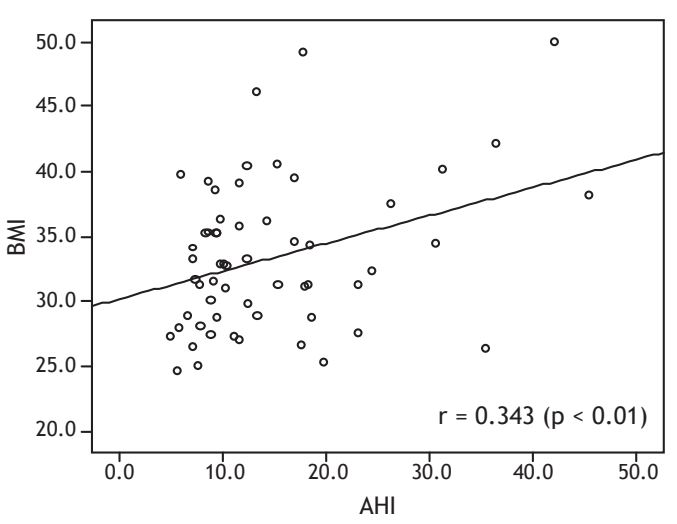

Figure 1. Correlation between the apnea-hypopnea index (AHI) and BMI in rapid-eye-movement-related obstructive sleep apnea.

REM-related OSA was higher among the women in our sample.

Various studies have shown that NREM-related OSA is more common among patients with high $\mathrm{AHI}$ values ( $\geq 30$ events/h). (15-17) Oksenberg et al. ${ }^{(18)}$ reported that, of their patients with NREM-related OSA, 49.1\% had severe OSA, similar to the $48.1 \%$ observed in our study. The effects that high NREM-AHI values (due to subnormal REM sleeping times) and sleeping in the supine position had on the overall $\mathrm{AHI}$ values were large, because NREM occupies a majority of the sleep time, even under normal conditions.

Despite the negative effects that sleeping in the supine position has on upper airway patency, many people prefer sleeping in that position. ${ }^{(19)}$ In patients

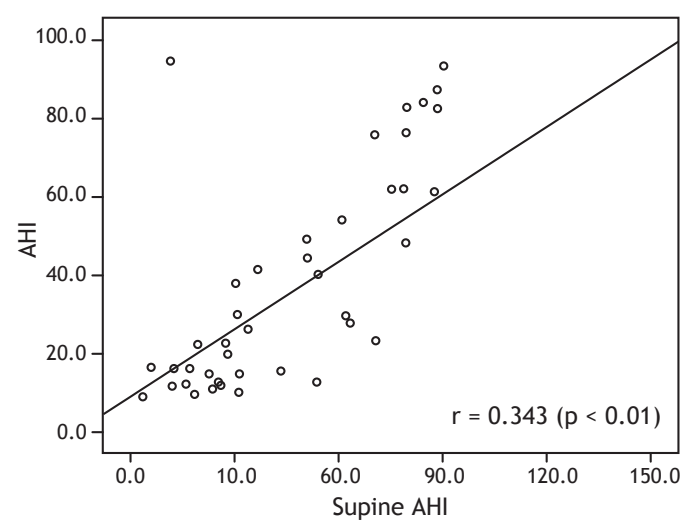

Figure 2. Correlation between the overall apnea-hypopnea index (AHI) and supine AHI in non-rapid-eye-movementrelated obstructive sleep apnea.

with positional OSA, the frequency and severity of respiratory events depend on how long the patient lies in the supine position. (20) During polysomnography, patients with OSA spend $46-51 \%$ of their total sleep time on their backs. (21) The supine position has been consistently associated with more severe OSA in adults. ${ }^{(22,23)}$ Sunnergren et al. also reported that the majority of subjects experienced more obstructive events when in the supine position than when in other positions. ${ }^{(24)}$ Sleep posture has different effects on REM sleep than on NREM sleep. ${ }^{25)}$ Specifically, the effects of sleeping in the supine and lateral positions differ between REM and NREM sleep, as reported by George et al. ${ }^{(6)}$ However, those authors found that the difference in AHI between the two sleep postures was much greater in patients NREM-related OSA. Pevernagie 
et al. reported that, among OSA patients, AHI values were higher in the supine position than in the lateral position only during NREM sleep. ${ }^{(25)}$ Cartwright et al. ${ }^{(26)}$ reported that OSA patients tend to sleep in the lateral position more often during REM sleep than during NREM sleep, a difference that we found to be significant in males but not in females. Previous studies have confirmed that women are more prone than are men to show higher AHI values during REM sleep than during NREM sleep, regardless of the sleep posture. ${ }^{(27)}$ In our study, s-AHI values were higher among men, whereas lateral AHI values were higher among women.

Patients with OSA experience fluctuations in oxygen levels during sleep. Sato et al. ${ }^{(28)}$ demonstrated that the drop in $\mathrm{SaO}_{2}$ is particularly dramatic in patients with severe OSA. A number of factors have been reported to affect the severity of oxygen desaturation during an apnea/hypopnea event, such factors including sleep posture, ${ }^{(29)}$ sleep stage, ${ }^{(30)}$ and age, ${ }^{(31)}$ as well as gender and obesity. ${ }^{(32)}$ In addition, comorbidity with COPD has been shown to increase the frequency and severity of oxygen desaturation in OSA. ${ }^{(33)}$ Bednarek et al. ${ }^{(34)}$ compared patients in whom OSA and COPD overlapped (i.e., patients with overlap syndrome) and patients with OSA only, in terms of polysomnographic variables. The authors reported that the patients in the overlap syndrome group had a lower mean oxygen saturation and spent more time in oxygen desaturation than did those in the OSA group.

Muraki et al.(17) found that, in OSA patients, the minimum $\mathrm{SaO}_{2}$ was significantly lower during REM sleep than during NREM sleep, as has been reported elsewhere. ${ }^{(3)}$ In the present study, although there were no differences between our two groups in terms of the minimum $\mathrm{SaO}_{2}$ or oxygen desaturation, the mean $\mathrm{SaO}_{2}$ was lower in the NREM-related OSA group than in the REM-related OSA group. We also found that, in REM-related OSA, oxygen desaturation correlated positively with age and BMI.

The termination of an apnea event is associated with arousal or awakening. Different levels or intensities of arousal can have quite different effects on sleep and breathing. ${ }^{(35)}$ In the present study, some of the effects of REM-related OSA were mitigated by a decrease in the time spent in REM sleep in parallel with increasing AHI-REM. This could be due to an increased number of events during REM sleep, leading to arousals and decreasing the time spent in REM sleep. In our study, there was no difference between the REM-related and NREM-related OSA groups, in terms of the number of arousals.

Punjabi et al.(13) found that the AHI-REM was associated with greater daytime sleepiness, whereas the AHI-NREM was not. However, Haba-Rubio et al., using an objective instrument (the Maintenance of Wakefulness Test), found no difference between patients with REM-related OSA and those with NREM-related OSA, in terms of excessive daytime sleepiness. ${ }^{(5)}$
Although OSA is a common chronic condition in all adults, its prevalence and severity are higher among men than among women. ${ }^{(29,36)}$ However, a number of studies have shown that REM-related OSA is more common among women. ${ }^{(7-9)}$ We find it interesting that the AHI-REM has been shown to be comparable between men and women. ${ }^{(37)}$ In our study, there was no significant difference between genders in terms of the prevalence of REM-related OSA. However, we found that REM-related OSA was more common than was NREM-related OSA among the women in our sample, whereas NREM-related OSA was more common among the men. In a study conducted by O'Connor et al., (27) OSA was found to be milder in the women than in the men. The authors reported that the significant respiratory events recorded in the women were associated with REM sleep and therefore concluded that REM-related OSA is more common in women.

Some studies have shown that REM-related OSA is more common among younger patients, ${ }^{(8)}$ whereas others have found no such age-related difference. ${ }^{(5,38)}$ In the present study, there was no significant difference between the REM-related and NREM-related OSA groups, in terms of age. However, in both groups, the women were older than were the men, although the female patients with REM-related OSA were younger than were those with NREM-related OSA, especially in the younger ( $<60$ year) age group. Koo et al. ${ }^{(7)}$ stated that, in REM-related and NREM-related OSA, the hormonal changes that occur with age in females have a protective effect against the respiratory problems that occur during NREM sleep. Among the women in our sample, REM-related OSA was more common than was NREM-related OSA, as well as being more common in the younger women.

In another study, Koo et al. showed that, with each passing decade of life, the NREM-AHI and REM-AHI increase by $11.2 \%$ and $9.0 \%$, respectively, in men, compared with $16.0 \%$ and $5.7 \%$, respectively, in women. ${ }^{(8)}$ The authors also showed that each 5-unit increase in BMI results in increases of $13.0 \%$ and $17.1 \%$ in the NREM-AHI and REM-AHI, respectively, in women, compared with a $24.2 \%$ decrease for both in men. ${ }^{(8)}$ In our study, there was no difference between the REM-related and NREM-related OSA groups in terms of the BMI. However, in both groups, the women were significantly heavier than were the men. We also found that, in the REM-related OSA group, the AHI showed a significant positive correlation with age and BMI. The AHI also correlated positively with BMI in the NREM-related OSA group, although the difference was not significant.

The present study has certain limitations. The first is that the study sample was evaluated retrospectively. Second, all of the polysomnography data analyzed for each patient were obtained during a single session of overnight polysomnography, as is customary in the clinical laboratory setting. Data collected over multiple nights of observation would provide important information regarding the impact of the differences in REM sleep and sleep posture, assuming that those 
parameters changed from night to night. A decrease in the proportion of REM sleep has been associated with the first night effect, and a total lack of REM sleep can occur in split-night polysomnography studies. ${ }^{(39)}$ Either of those scenarios could lead to an underestimation of $\mathrm{AHI}$ in patients with REM-related OSA.

In conclusion, it appears that REM-related OSA is more common among patients with mild-to-moderate OSA, whereas NREM-related OSA is more common among those with severe OSA, the latter being associated mainly with s-AHI. Our findings indicate that s-AHI has a more significant effect on the severity of OSA than does REM-AHI. When determining OSA severity and choosing among treatment modalities, physicians should take into consideration not only sleep stage but also sleep posture.

\section{REFERENCES}

1. Fenik VB, Davies RO, Kubin L. REM sleep-like atonia of hypoglossal (XII) motoneurons is caused by loss of noradrenergic and serotonergic inputs. Am J Respir Crit Care Med. 2005;172(10):1322-30. http:// dx.doi.org/10.1164/rccm.200412-17500C

2. Conwell W, Patel B, Doeing D, Pamidi S, Knutson KL, Ghods F, et al. Prevalence, clinical features, and CPAP adherence in REM-related sleep-disordered breathing: a cross-sectional analysis of a large clinical population. Sleep Breath. 2012;16(2):519-26. http://dx.doi. org/10.1007/s11325-011-0537-6

3. Findley LJ, Wilhoit SC, Suratt PM. Apnea duration and hypoxemia during REM sleep in patients with obstructive sleep apnea. Chest. 1985;87(4):432-6. http://dx.doi.org/10.1378/chest.87.4.432

4. Quera-Salva MA, Guilleminault $C$, Partinen $M$, Jamieson A Determinants of respiratory disturbance and oxygen saturation drop indices in obstructive sleep apnoea syndrome. Eur Respir J 1988; 1(7):626-31.

5. Haba-Rubio J, Janssens JP, Rochat T, Sforza E. Rapid eye movementrelated disordered breathing: clinical and polysomnographic features. Chest. 2005;128(5):3350-7. http://dx.doi.org/10.1378/ chest.128.5.3350

6. George CF, Millar TW, Kryger MH. Sleep apnea and body position during sleep. Sleep. 1988;11(1):90-9.

7. Koo BB, Dostal J, loachimescu O, Budur K. The effects of gender and age on REM-related sleep-disordered breathing. Sleep Breath. 2008;12(3):259-64. http://dx.doi.org/10.1007/s11325-007-0161-7

8. Koo BB, Patel SR, Strohl K, Hoffstein V. Rapid eye movement-related sleep-disordered breathing: influence of age and gender. Chest. 2008;134(6):1156-61. http://dx.doi.org/10.1378/chest.08-1311

9. Resta O, Carpanano GE, Lacedonia D, Di Gioia G, Giliberti T, Stefano $A$, et al. Gender difference in sleep profile of severely obese patients with obstructive sleep apnea (OSA). Respir Med. 2005;99(1):91-6. http://dx.doi.org/10.1016/j.rmed.2004.05.014

10. Iber C Ancoli-Israel S, Chesson A, Quan SF. The AASM Manual for the Scoring of Sleep and Associated Events: Rules, Terminology and Technical Specifications. Westchester, IL: American Academy of Sleep Medicine; 2007

11. Berry RB, Budhiraja R, Gottlieb DJ, Gozal D, Iber C, Kapur VK, et al. Rules for scoring respiratory events in sleep: update of the 2007 AASM Manual for the Scoring of Sleep and Associated Events. Deliberations of the Sleep Apnea Definitions Task Force of the American Academy of Sleep Medicine. J Clin Sleep Med. 2012;8(5):597-619.

12. Johns MW. A new method for measuring daytime sleepiness: The Epworth sleepiness scale. Sleep. 1991;14(6):540-5

13. Punjabi NM, Bandeen-Roche K, Marx JJ, Neubauer DN, Smith $\mathrm{PL}$, Schwartz AR. The association between daytime sleepiness and sleep-disordered breathing in NREM and REM sleep. Sleep. 2002;25(3):307-14

14. Campos-Rodríguez F, Fernández-Palacín A, Reyes-Núñez N, ReinaGonzález A. Clinical and polysomnographic features of rapid-eyemovement-specific sleep-disordered breathing [Article in Spanish] Arch Bronconeumol. 2009;45(7):330-4. http://dx.doi.org/10.1016/1. arbres.2008.12.003

15. Kutbay Özçelik H, Akkoyunlu ME, Bostanlı P, Bayram M, Atahan E, et al. The frequency and properties of REM related obstructive sleep apnea among the patients with mild related obstructive sleep apnea [Article in Turkish]. Tuberk Toraks. 2013:61(4):283-7. http://dx.doi. org/10.5578/tt.6208

16. Liu $Y$, Su C, Liu R, Lei G, Zhang W, Yang T, et al. NREM-AHI greater than REM-AHI versus REM-AHI greater than NREM-AHI in patients with obstructive sleep apnea: clinical and polysomnographic features. Sleep Breath. 2011;15(3):463-70. http://dx.doi.org/10.1007/ s11325-010-0358-z

17. Muraki M, Kitaguchi S, Ichihashi H, Haraguchi R, Iwanaga T, Kubo H et al. Apnoea-hypopnoea index during rapid eye movement and nonrapid eye movement sleep in obstructive sleep apnoea. J Int Med Res. 2008;36(5):906-13. http://dx.doi.org/10.1177/147323000803600506

18. Oksenberg A, Arons E, Nasser K, Vander T, Radwan H. REM-related obstructive sleep apnea: the effect of body position. J Clin Sleep Med. 2010;6(4):343-8.

19. Oksenberg A, Silverberg DS, Arons E, Radwan H. Positional vs nonpositional obstructive sleep apnea patients: anthropomorphic nocturnal polysomnographic, and multiple sleep latency test data. Chest. 1997;112(3):629-39. http://dx.doi.org/10.1378/ chest.112.3.629

20. Dempsey JA, Veasey SC, Morgan BJ, O'Donnell CP. Pathophysiology of sleep apnea. Physiol Rev. 2010;90(1):47-112. http://dx.doi. org/10.1152/physrev.00043.2008

21. Metersky ML, Castriotta RJ. The effect of polysomnography on sleep position: possible implications on the diagnosis of positional obstructive sleep apnea. Respiration. 1996;63(5):283-7. http://dx.doi. org/10.1159/000196561

22. Menon A, Kumar M. Influence of body position on severity of obstructive sleep apnea: a systematic review. ISRN Otolaryngol. 2013;2013:670381. http://dx.doi.org/10.1155/2013/670381

23. Eiseman NA, Westover MB, Ellenbogen JM, Bianchi MT. The impact of body posture and sleep stages on sleep apnea severity in adults. J Clin Sleep Med. 2012;8(6):655-66A. http://dx.doi.org/10.5664/ jcsm.2258

24. Sunnergren O, Broström A, Svanborg E. Positional sensitivity as a confounder in diagnosis of severity of obstructive sleep apnea. Sleep Breath. 2013;17(1):173-9. http://dx.doi.org/10.1007/s11325-0120666-6

25. Pevernagie DA, Slanson AW, Sheedy PF 2nd, Daniels BK, Shepard JW Jr. Effects of body position on the upper airway of patients with obstructive sleep apnea. Am J Respir Crit Care Med. 1995;152(1):17985. http://dx.doi.org/10.1164/ajrccm.152.1.7599821

26. Cartwright Rd, Diaz F, Lloyd S. The effects of sleep posture and sleep stage on apnea frequency. Sleep. 1991;14(4):351-3.

27. O'Connor C, Thornley KS, Hanly PJ. Gender differences in the polysomnographic features of obstructive sleep apnea. Am J Respir Crit Care Med. 2000;161(5):1465-72. http://dx.doi.org/10.1164/ ajrccm.161.5.9904121

28. Sato M, Suzuki M, Suzuki J, Endo $Y$, Chiba $Y$, Matsuura $M$, et al. Overweight patients with severe sleep apnea experience deeper oxygen desaturation at apneic events. J Med Dent Sci. 2008;55(1):43 7.

29. Oksenberg A, Khamaysi I, Silverberg DS, Tarasiuk A. Association of body position with severity of apneic events in patients with severe nonpositional obstructive sleep apnea. Chest. 2000;118(4):1018-24. http://dx.doi.org/10.1378/chest.118.4.1018

30. Sériès F, Cormier Y, La Forge J. Influence of apnea type and sleep stage on nocturnal postapneic desaturation. Am Rev Respir Dis. 1990;141(6):1522-6. http://dx.doi.org/10.1164/ajrccm/141.6.1522

31. George E, Katerina V, Maria S, Lambros B, Konstantina N, Dimitrios G. Clinical features and polysomnographic findings in Greek male patients with obstructive sleep apnea syndrome: differences regarding the age. Sleep Disord. 2012;2012:324635. http://dx.doi. org/10.1155/2012/324635

32. Peppard PE, Ward NR, Morrell MJ. The impact of obesity on oxygen desaturation during sleep-disordered breathing. Am J Respir Crit Care Med. 2009:180(8):788-93. http://dx.doi.org/10.1164/rccm.20090507730C 
Obstructive sleep apnea related to rapid-eye-movement or non-rapid-eye-movement sleep:

comparison of demographic, anthropometric, and polysomnographic features

33. Marin JM, Soriano JB, Carrizo SJ, Boldova A, Celli BR. Outcomes in patients with chronic obstructive pulmonary disease and obstructive sleep apnea: the overlap syndrome. Am J Respir Crit Care Med. 2010;182(3):325-31. http://dx.doi.org/10.1164/rccm.200912-18690C

34. Bednarek M, Plywaczewski R, Jonczak L, Zielinski J. There is no relationship between chronic obstructive pulmonary disease and obstructive sleep apnea syndrome: a population study. Respiration. 2005;72(2):142-9. http://dx.doi.org/10.1159/000084044

35. Farney RJ, Walker LE, Jensen RL, Walker JM. Ear oximetry to detect apnea and differentiate rapid eye movement (REM) and non-REM (NREM) sleep. Screening for the sleep apnea syndrome. Chest. 1986;89(4):533-9. http://dx.doi.org/10.1378/chest.89.4.533

36. Catcheside PG, Jordan A. Reflex tachycardia with airway opening in obstructive sleep apnea. Sleep. 2013;36(6):819-21. http://dx.doi org/10.5665/sleep.2698

37. Martinez D, Lumertz MS, Lenz Mdo C. Dimensions of sleepiness and their correlations with sleep-disordered breathing in mild sleep apnea. J Bras Pneumol. 2009;35(6):507-14. http://dx.doi.org/10.1590/ S1806-37132009000600003

38. Su CS, Liu KT, Panjapornpon K, Andrews N, Foldvary-Schaefer N Functional outcomes in patients with REM-related obstructive sleep apnea treated with positive airway pressure therapy. J Clin Sleep Med. 2012;8(3):243-7. http://dx.doi.org/10.5664/jcsm.1902

39. Toussaint $M$, Luthringer $R$, Schaltenbrand $N$, Nicolas $A$, Jacamin $A$ Carelli $\mathrm{G}$, et al. Changes in EEG power density during sleep laboratory adaptation. Sleep. 1997;20(12):1201-7. 